\title{
Does SARS-CoV-2 Bind to Human ACE2 Stronger than SARS-CoV?
}

Hoang Linh Nguyen ${ }^{1,+}$, Pham Dang Lan ${ }^{1,2,+}$, Nguyen Quoc Thai ${ }^{1,3,+}$, Daniel A. Nissley ${ }^{4}$, Edward P. $\mathrm{O}^{\prime} \mathrm{Brien}^{5,6,7}$, and Mai Suan $\mathrm{Li}^{8, *}$

${ }^{1}$ Life Science Lab, Institute for Computational Science and Technology, Quang Trung

Software City, Tan Chanh Hiep Ward, District 12, Ho Chi Minh City, Vietnam

${ }^{2}$ Faculty of Physics and Engineering Physics, VNUHCM-University of Science, 227, Nguyen

Van Cu Street, District 5, Ho Chi Minh City, Vietnam

${ }^{3}$ Dong Thap University, 783 Pham Huu Lau Street, Ward 6, Cao Lanh City, Dong Thap,

Vietnam

${ }^{4}$ Department of Statistics, University of Oxford, Oxford, United Kingdom

${ }^{5}$ Department of Chemistry, Penn State University, University Park, Pennsylvania, United States

${ }^{6}$ Bioinformatics and Genomics Graduate Program, The Huck Institutes of the Life Sciences, Penn State University, University Park, Pennsylvania, United States

${ }^{7}$ Institute for Computational and Data Sciences, Penn State University, University Park, Pennsylvania, United States

${ }^{8}$ Institute of Physics, Polish Academy of Sciences, al. Lotnikow 32/46, 02-668, Warsaw, Poland

${ }^{+}$These authors contributed equally

*Email: masli@ifpan.edu.pl; Phone: +48 886813018

\section{Supporting Information for "Does SARS-CoV-2 Bind to Human ACE2 Stronger than SARS-CoV?"}



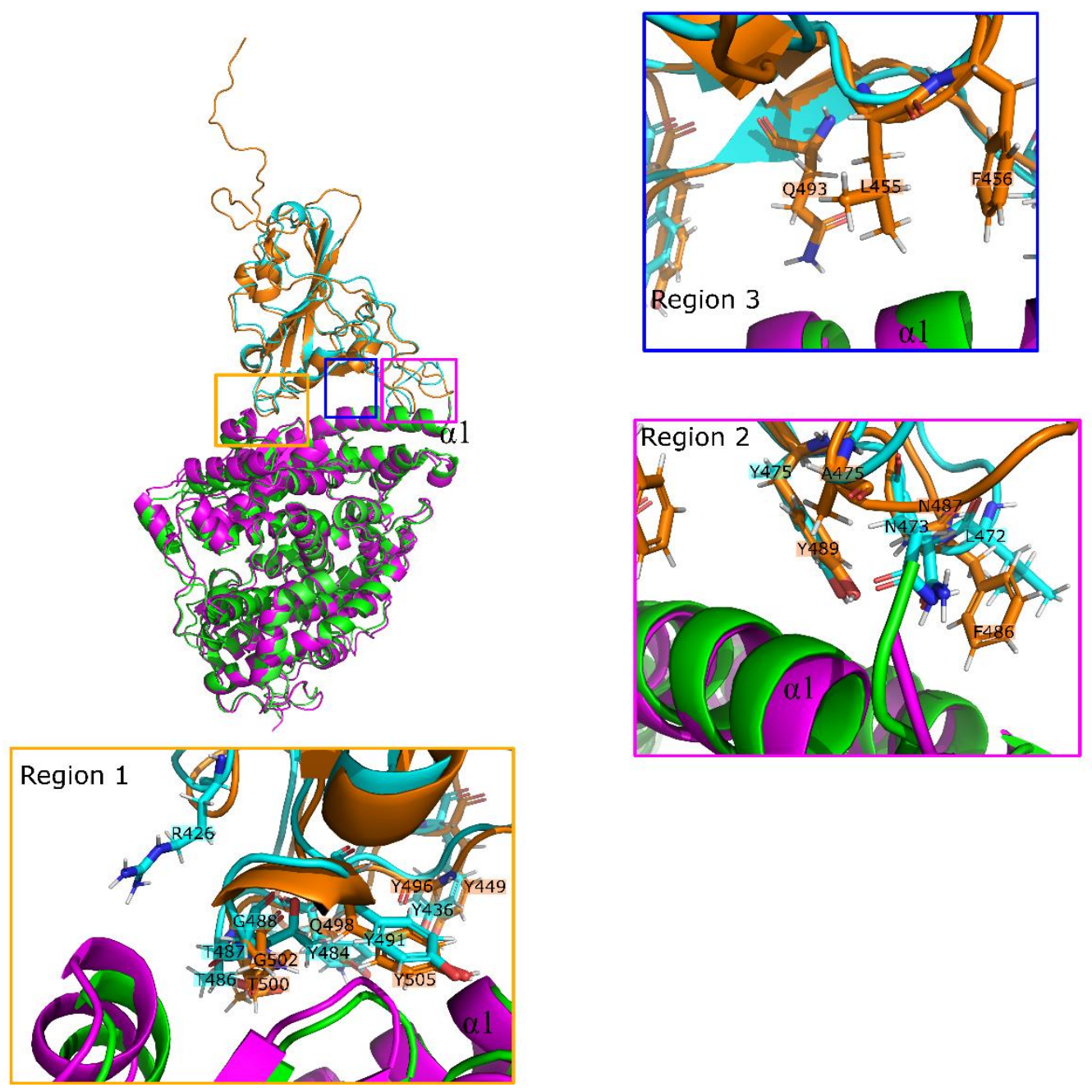

Figure S1: Superimposition of the initial structures of two complexes. The first two lines are a sequence alignment between SARS-CoV and SARS-CoV-2 for the three boxed regions shown below. ACE2-PD in complex with SARS-CoV-RBD and SARS-CoV-2-RBD is highlighted in magenta and green, respectively, and SARS-CoV-RBD and SARS-CoV-2-RBD, cyan and orange, respectively. The residues of the RDB spike proteins that form $\mathrm{HB}$ and $\mathrm{NBC}$ with ACE2-PD are shown in color boxes. 


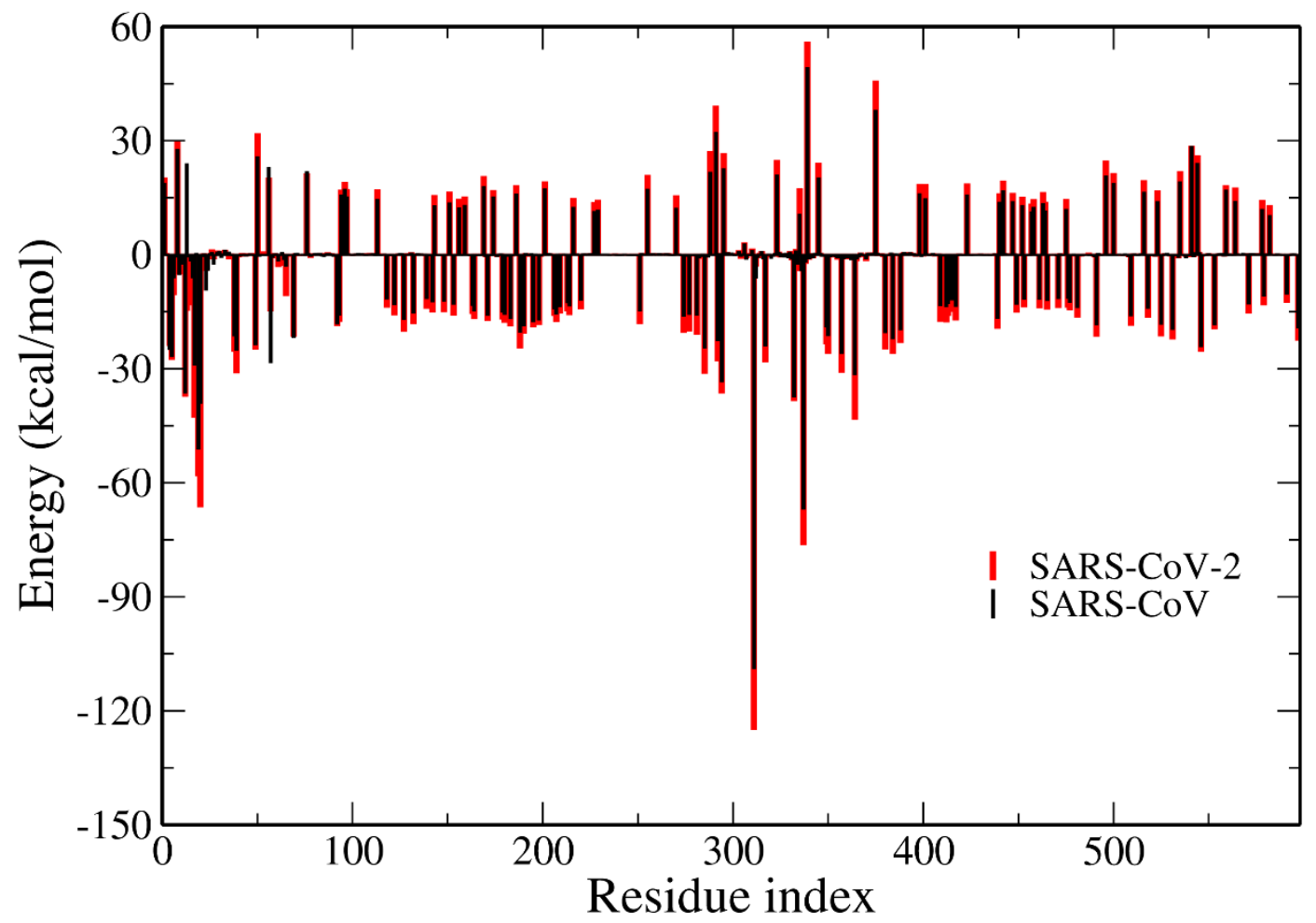

Figure S2: Contributions of the residues of ACE2-PD to the non-bonded interaction energy $(\mathrm{kcal} / \mathrm{mol})$. Results were obtained by averaging over 5 trajectories and a window of 0-7000 ns, pulling speed $v=0.5 \mathrm{~nm} / \mathrm{s}$
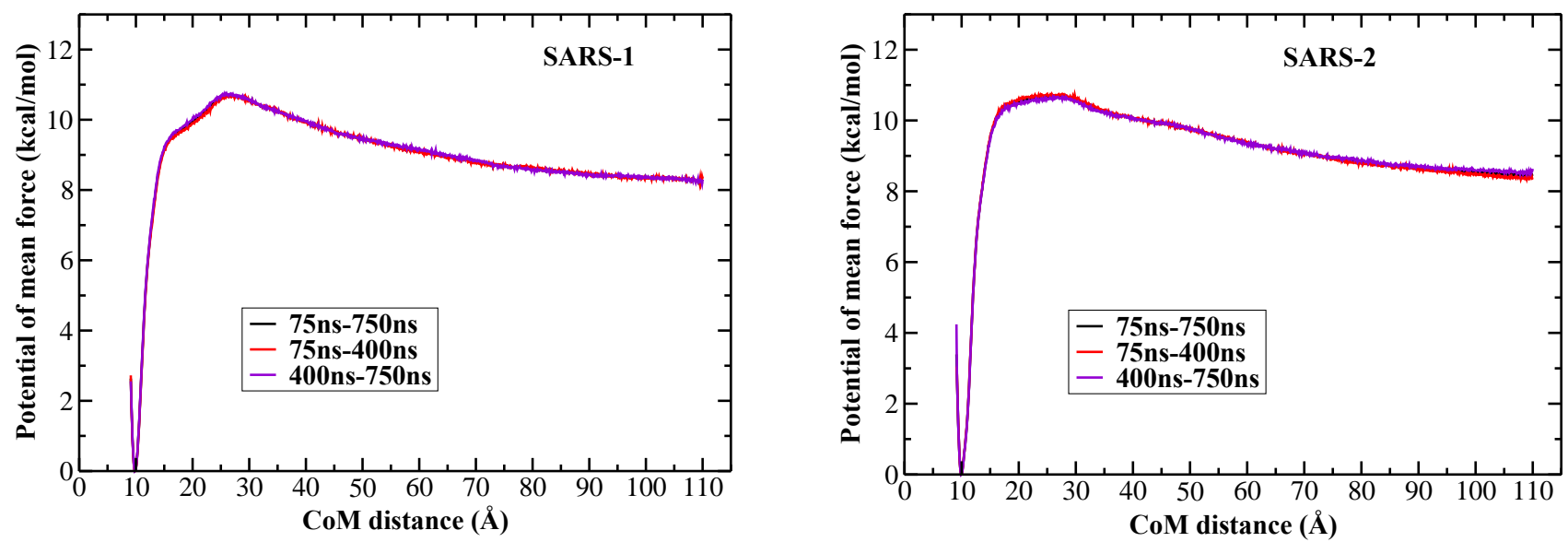

Figure S3: Convergence of REX-US simulations of SARS-Cov (left panel) and SARS-CoV-2 (right panel) through 1D-PMF profiles corresponding to different time windows. 